\title{
Spectropolarimetry of the Type Ia SN 2019ein rules out significant global asphericity of the ejecta
}

\author{
Kishore C. Patra ${ }^{1, \dagger, \star}$, Yi Yang (杨轶) ${ }^{1,2, \S, \dagger}$, Thomas G. Brink ${ }^{1}$, Peter Höflich ${ }^{3}$, Lifan Wang ${ }^{4}$, \\ Alexei V. Filippenko ${ }^{1,5, \$}$, Daniel Kasen ${ }^{1,6}$, Dietrich Baade ${ }^{7}$, Ryan J. Foley ${ }^{8}$, Justyn R. Maund ${ }^{9}$, \\ WeiKang Zheng ${ }^{1}$, Tiara Hung ${ }^{8}$, Aleksandar Cikota ${ }^{10}$, J. Craig Wheeler ${ }^{11}$, Mattia Bulla ${ }^{12}$ \\ ${ }^{1}$ Department of Astronomy, University of California, Berkeley, CA 94720-3411, USA \\ ${ }^{2}$ Department of Particle Physics and Astrophysics, Weizmann Institute of Science, Rehovot 76100, Israel \\ ${ }^{3}$ Department of Physics, Florida State University, 77 Chieftan Way, Tallahassee, FL 32306, USA \\ ${ }^{4}$ George P. and Cynthia Woods Mitchell Institute for Fundamental Physics \& Astronomy, Texas A\&M University, 4242 TAMU, College Station, TX 77843, USA \\ ${ }^{5}$ Miller Institute for Basic Research in Science, University of California, Berkeley, CA 94720, USA \\ ${ }^{6}$ Departments of Physics and Astronomy, University of California, Berkeley; and Lawrence Berkeley National Laboratory, USA \\ ${ }^{7}$ European Organisation for Astronomical Research in the Southern Hemisphere (ESO), Karl-Schwarzschild-Str. 2, 85748 Garching b. München, Germany \\ ${ }^{8}$ Department of Astronomy and Astrophysics, University of California, Santa Cruz, CA 95064, USA \\ ${ }^{9}$ Department of Physics and Astronomy, University of Sheffield, Hicks Building, Hounsfield Road, Sheffield S3 7RH, UK \\ ${ }^{10}$ European Southern Observatory, Alonso de Córdova 3107, Vitacura, Casilla 19001, Santiago de Chile, Chile \\ ${ }^{11}$ Department of Astronomy, University of Texas, Austin, TX 78712, USA \\ ${ }^{12}$ The Oskar Klein Centre, Department of Astronomy, Stockholm University, AlbaNova, SE-10691 Stockholm, Sweden \\ ${ }^{\dagger}$ Nagaraj-Noll Graduate Fellow \\ $\S_{\text {Bengier-Winslow-Robertson Fellow }}$ \\ ${ }^{\ddagger}$ Miller Senior Fellow
}

Accepted XXX. Received YYY; in original form ZZZ

\begin{abstract}
Detailed spectropolarimetric studies may hold the key to probing the explosion mechanisms and the progenitor scenarios of Type Ia supernovae (SNe Ia). We present multi-epoch spectropolarimetry and imaging polarimetry of SN 2019ein, an SN Ia showing high expansion velocities at early phases. The spectropolarimetry sequence spans from $\sim-11$ to +10 days relative to peak brightness in the $B$-band. We find that the level of the continuum polarization of SN 2019ein, after subtracting estimated interstellar polarization, is in the range $0.0-0.3 \%$, typical for SNe Ia. The polarization position angle remains roughly constant before and after the SN light-curve peak, implying that the inner regions share the same axisymmetry as the outer layers. We observe high polarization ( 1\%) across both the Si II $\lambda 6355$ and $\mathrm{Ca}$ II near-infrared triplet features. These two lines also display complex polarization modulations. The spectropolarimetric properties of SN 2019ein rule out a significant departure from spherical symmetry of the ejecta for up to a month after the explosion. These observations disfavour merger-induced and double-detonation models for SN 2019ein. The imaging polarimetry shows weak evidence for a modest increase in polarization after $\sim 20$ days since the $B$-band maximum. If this rise is real and is observed in other SNe Ia at similar phases, we may have seen, for the first time, an aspherical interior similar to what has been previously observed for SNe IIP. Future polarization observations of SNe Ia extending to post-peak epochs will help to examine the inner structure of the explosion.
\end{abstract}

Key words: polarization - supernovae: individual (SN 2019ein) - techniques: polarimetric - white dwarfs

\section{INTRODUCTION}

During the last half century, Type Ia supernovae (SNe Ia; see Filippenko 1997 and Gal-Yam 2017 for reviews of SNe) have answered (and posed) a myriad of interesting questions in astrophysics. These range from nucleosynthesis, chemical enrichment (Renzini 1999), and heating of the interstellar medium (Ciotti et al. 1991) to the discovery of the accelerating expansion of the universe (Riess et al.

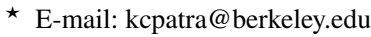

$\dagger$ E-mail: yi.yang@berkeley.edu
}

1998; Perlmutter et al. 1999), and more recently the so-called "Hubble tension" (as summarised by Riess 2020). Yet, the nature of the progenitor systems of SNe Ia is still unclear. It has been generally established that the rise of SNe Ia is powered by the thermonuclear runaway of white dwarfs (WDs; see Hoyle \& Fowler 1960; Howell 2011; Hillebrandt et al. 2013; Maoz et al. 2014; Höflich 2017; Soker 2019 for recent reviews). However, the exact mechanism by which a WD's explosion is triggered and propagates through the progenitor still remains poorly understood (Arnett 1969; Nomoto et al. 1976; Khokhlov 1991; Niemeyer et al. 1996; Reinecke et al. 2002; Plewa et al. 2004; Röpke 2007; Pakmor et al. 2011; Seitenzahl et al. 2013). 
Multiple channels of progenitors have been theorised, including the double-degenerate scenario in which two WDs merge (Iben \& Tutukov 1984; Webbink 1984), the single-degenerate scenario in which a WD accretes matter from a nondegenerate companion until the Chandrasekhar mass $\left(M_{\mathrm{Ch}} \approx 1.4 M_{\odot}\right)$ is approached (Whelan \& Iben 1973), and tidal disruption of a WD by a compact companion and subsequent detonation of the WD (Rosswog et al. 2009; Luminet \& Pichon 1989).

Among these progenitor scenarios, a range of explosion mechanisms might be possible: delayed detonation, in which an initial deflagration front transitions to a detonation in a WD (Khokhlov 1991); double detonation, where a thin He layer on the WD detonates first, starting a detonation front in the WD (see, for example, Fink et al. 2010; Taam 1980; Shen et al. 2010); and compressional heating of WDs triggered by the dynamic merger of two C-O WDs (Pakmor et al. 2010; Hayden et al. 2010) or head-on collisions of WDs (Kushnir et al. 2013).

The shape of the ejecta and their circumstellar configuration is spatially unresolvable for extragalactic SN explosions, even with the best of ground-based interferometers ${ }^{1}$. Conventional photometry and spectroscopy provide a way to probe the kinematics and chemical structures of SN ejecta and their interaction with any pre-explosion circumstellar matter (CSM; see, for example, Nugent et al. 2011; GalYam et al. 2014). However, these observations only offer crude clues on the structures of the ejecta and the interaction between the ejecta and any existing CSM. Such information is projected and smeared into the single dimension of radial velocity. Fortunately, spectropolarimetry, which measures polarization as a function of wavelength, provides a unique approach to the study of the SN explosion geometry. Any asphericity of the SN ejecta and the distribution of various elements formed in the ejecta are traced by the level of the continuum and the profiles of associated spectral lines in the polarization spectra, respectively. Additionally, the footprint of the interaction between the SN ejecta with any companion and CSM is encoded in the polarization spectra since such processes may create non-sphericallysymmetric emission and/or scattering photon sources.

In $\mathrm{SN}$ atmospheres, photons are scattered by free electrons (Thomson scattering). The polarization state of the emitted photons is determined when they escape the last-scattering surface, known as the photosphere. A photon that undergoes Thomson scattering will be polarized perpendicularly to the plane of scattering, which is defined as the plane containing the incident and scattered rays. For a spatially-unresolved source, the observed polarization is an integration of the photons' electric vectors (E-vectors) projected in the plane of the sky. If the projected photosphere is circularly symmetric, a complete cancellation of the E-vectors results in zero net polarization. However, if the projected photosphere deviates from circular symmetry, incomplete cancellation of the E-vectors would lead to nonzero polarization across the spectrum. Additionally, any clumps of high-opacity absorbing material present above the photosphere along the observer's line of sight may block parts of the underlying photosphere. Therefore, an incomplete cancellation of the E-vectors will occur across the corresponding spectral lines, further producing nonzero polarization at the extinguished wavelengths.

SN 2019ein $[\alpha(\mathrm{J} 2000)=13: 53: 29.11, \delta(\mathrm{J} 2000)=+40: 16: 31.33]$ was discovered on 2019 May 1.47 (UT dates are used throughout this paper; Tonry et al. 2019) in the outskirts of the nearby galaxy NGC

\footnotetext{
1 The minimum resolution required to study a nearby SN, for instance $3 \mathrm{Mpc}$ away with a photosphere 100 au wide, would be $\sim 10 \mu$ as. For comparison, the Event Horizon Telescope can achieve a resolution of $\sim 60 \mu$ as.
}

5353. The host of SN 2019ein is a lenticular galaxy (Hubble type S0). A redshift of $z=0.00775$ taken from the NASA/IPAC Extragalactic Database $^{2}$ was adopted in this study. The spectrum obtained by the Las Cumbres Observatory (LCO) Global SN project on 2021 May 2.3 (about two weeks before the $B$-band light-curve peak) shows a very high expansion velocity of $\sim 24,000 \mathrm{~km} \mathrm{~s}^{-1}$ as inferred from the absorption minimum of the Si II $\lambda 6355$ line. The Ca II near-

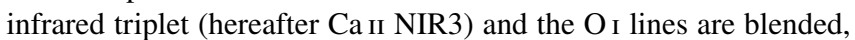
creating a broad absorption profile. Curiously, the entire spectrum was slightly blueshifted with respect to the host-galaxy redshift, with the emission peaks of $\mathrm{Si}$ II, Ca II, and $\mathrm{S}_{\text {II }}$ exhibiting velocities $10,000 \mathrm{~km} \mathrm{~s}^{-1}$ toward the observer. Pellegrino et al. (2020) suggested that the apparent blueshift may be caused by an asymmetric explosion resulting in enhanced abundance of material at high velocities or due to optical-depth effects in the photosphere, in which most of the flux comes from material moving along the SN-Earth line of sight. The rise time of SN 2019ein was short (15.37 \pm 0.55 days), after which the SN faded rapidly with a 15 day post-peak $B$-band magnitude decline (Phillips 1993) $\Delta m_{15}(B)=1.36 \pm 0.02 \mathrm{mag}$ (Kawabata et al. 2020).

These features put SN2019ein in the rare company of highvelocity SNe Ia like SNe 2004dt and 2006X, for which spectropolarimetric data have been obtained (Wang et al. 2006; Patat et al. 2009). In this work, we present five epochs of spectropolarimetry of SN 2019ein. We describe our observations in Section 2 and present our results along with the analysis in Section 3. We discuss the interpretations of the data in Section 4 and provide a concluding summary in Section 5.

\section{OBSERVATIONS AND DATA REDUCTION}

\subsection{Kast Spectropolarimetry}

Spectropolarimetry of SN 2019ein was obtained using the polarimetry mode of the Kast double spectrograph on the Shane $3 \mathrm{~m}$ telescope at Lick Observatory (Miller et al. 1988). In the polarimetry mode, the light beam incident on the spectrograph is passed through a rotatable, half-wave plate and then through a Wollaston Prism. The prism splits the incident light into two perpendicularly polarized components, named the ordinary and the extraordinary beams, which appear on the detector as two parallel traces. Only the red channel of Kast was used for spectropolarimetry. A GG455 order-sorting filter was in place, blocking all first-order light below $\sim 4600 \AA$ and all secondorder light below $\sim 9800 \AA$. The usable wavelength range of the setup was 4600-9000 ̊. Observations were made with the 300 lines $\mathrm{mm}^{-1}$ grating and the $3^{\prime \prime}$-wide slit, resulting in a resolution of $\Delta \lambda \approx 18 \AA$ $\left(\sim 800 \mathrm{~km} \mathrm{~s}^{-1}\right)$ at the central wavelength $\sim 6800 \AA$.

Flatfield and arc-lamp exposures were obtained at the beginning of the observation night. The flatfield spectra were produced by the reflection of the light from an incandescent lamp off of the inner surface of the dome.

SN 2019ein and polarization standard stars were observed each night. Four exposures were carried out at retarder-plate angles of $0^{\circ}, 45^{\circ}, 22.5^{\circ}$, and $67.5^{\circ}$. Multiple sets of polarimetry exposures were obtained for SN 2019ein to achieve higher signal-to-noise ratios $(\mathrm{S} / \mathrm{N})$. Since all observations were carried out at small airmasses ( $\lesssim 1.25$; see Table 1 ), we aligned the slit to the position angle of $180^{\circ}$ (north-south direction). Because Kast does not have an atmospheric dispersion compensator (ADC) to atone for the differential loss of blue light (Filippenko 1982), the following sanity check was carried

\footnotetext{
2 https://ned.ipac.caltech.edu
} 
out. For each night, we compared the Stokes parameters measured for different sets of spectropolarimetry with the values derived for the set obtained at the smallest airmass, typically 1.05. The Stokes parameters for each set were consistent within the associated uncertainties, suggesting a negligible effect on the polarization measurement from the loss of blue light.

Our nightly observations of the unpolarized standard star HD 110897 confirmed the low instrumental polarization of the Kast spectrograph (Sec. 3.2). The polarizance test to determine the instrumental response to $100 \%$ linearly polarized light was done by observing the same unpolarized standard star through a polarizing filter. Each night we also conducted spectropolarimetry of two polarization standard stars chosen among HD 154445, HD 161056, and HD 155528 to determine the accuracy of polarimetric measurements (see Sec. 3.2 for more details). A polarization "probe star" was also observed to estimate the Galactic interstellar polarization (see Sec. 3.3).

Extraction of the ordinary and the extraordinary beams was carried out following standard techniques for CCD processing and spectrum extraction within $\mathrm{IRAF}^{3}$. The images were bias subtracted using an overscan region. Cosmic ray hits on the detector were removed with L.A.Cosmic (van Dokkum 2001). For each night, flatfield images were combined and normalised by a low-order spline function to fit the continuum before applying to the science images. Then we used the prescription of Horne (1986) to optimally extract each spectrum independently from the science images with apertures typically set to width at $\sim 1-2 \%$ of the maximum of the spectrum profile. The background apertures were usually $5-10$ pixels wide and placed 2-3 times the value of the full width at half-maximum intensity (FWHM) away from the profile centre.

Wavelength calibration was conducted separately for the ordinary and extraordinary beams in each individual exposure (all four retarder-plate angles) using lamp exposures. Small wavelength adjustments determined from the night-sky lines in the object frames were also applied to fine tune the wavelength calibration. A typical root-mean-square (RMS) accuracy of $\sim 0.2 \AA$ was achieved. Flux calibration of the ordinary and extraordinary beams of the SN was applied using the corresponding beam of a flux standard star observed at a similar airmass. We fit splines to the continuum of the flux-standard spectrum to generate a "sensitivity function" that maps CCD counts to the flux at each wavelength. This mapping function was then applied to the SN spectra. Correction for telluric absorption was carried out by interpolating over the atmospheric absorption regions of the flux-standard spectrum.

\subsection{RINGO3 Imaging Polarimetry}

In this work, we adopted the imaging polarimetry of SN2019ein from Maund et al. (2021). Details of the RINGO3 observations and data reduction are provided by Maund et al. (2021). In brief, they obtained the data using the Liverpool Telescope (LT) located on the Canary Island of La Palma, with the RINGO3 polarimeter (Arnold et al. 2012). Observations were carried out in three cameras optimised to integrate over the following wavelength ranges: $b, 3500-6400 \AA$; $g, 6500-7600 \AA$; and $r, 7700-10000 \AA$. Interstellar polarization was subtracted from the Stokes parameters measured by Maund et al.

\footnotetext{
3 IRAF is distributed by the National Optical Astronomy Observatories, which are operated by the Association of Universities for Research in Astronomy, Inc., under cooperative agreement with the National Science Foundation (NSF).
}

(2021) (see Sec. 3.7 for details). The Stokes parameters, polarization, and its position angle are shown in Table 2.

\section{ANALYSIS AND RESULTS}

\subsection{Calculating the Stokes $q$ and $u$}

We express the normalised Stokes parameters as $q=Q / I$ and $u=U / I$, where $Q$ and $U$ are the differences in flux with E-vector oscillating in two perpendicular directions and $I$ is the total flux. $U$ measures polarization along angles that are rotated by $45^{\circ}$ with respect to those measured by $Q$.

We calculate $q$ and $u$ from two sets of spectra obtained with the waveplate at $\left[0^{\circ}, 45^{\circ}\right]$, and $\left[22^{\circ} .5,67^{\circ} .5\right]$, respectively. From the ordinary $(o)$ and the extraordinary $(e)$ flux beams $(f), q$ can be expressed as

$q_{o}=\frac{f_{o, 0}-f_{o, 45}}{f_{o, 0}+f_{o, 45}}$ and $q_{e}=\frac{f_{e, 0}-f_{e, 45}}{f_{e, 0}+f_{e, 45}}$,

respectively, which are then averaged. Similarly, we calculate $u$ using the exposures at the other set of waveplate positions. The observed polarization is then given by

$p_{\mathrm{obs}}=\sqrt{q^{2}+u^{2}}$

and the polarization position angle $(P A)$ is

$P A_{\mathrm{obs}}=\frac{1}{2} \arctan \left(\frac{u}{q}\right)$.

The polarization defined this way is positive-definite and biased toward higher polarization, especially in the low $\mathrm{S} / \mathrm{N}$ regime. The final derived polarization was achieved after a debiasing procedure following Wang et al. (1997):

$p=\left(p_{\mathrm{obs}}-\frac{\sigma_{p}^{2}}{p_{\mathrm{obs}}}\right) \times h\left(p_{\mathrm{obs}}-\sigma_{p}\right)$ and $P A=P A_{\mathrm{obs}}$,

where $\sigma_{p}$ denotes the $1 \sigma$ uncertainty in $p$ and $h$ is the Heaviside step function. The flux spectrum is calculated by averaging all the spectra of $o$-rays and $e$-rays used in deriving $q$ and $u$. Figures 1-5 show the measured Stokes $q, u, p, P A$, and total flux at each epoch.

\subsection{Polarimetric Calibration}

The nightly-measured Stokes $q$ and $u$ of the unpolarized standard star HD 110897 are consistent with a level of $<0.05 \%$, indicating a low instrumental polarization and a high stability of the Kast spectropolarimeter over time. In the polarizance test with the same standard star, we determined that the polarimetric response of the instrument is larger than $99.5 \%$ across the entire wavelength range (4600-9000 $\mathrm{A}$ ) and therefore does not necessitate further correction. The polarization position angle of SN 2019ein was corrected as follows:

$$
\begin{gathered}
q_{\mathrm{corr}}=p_{\mathrm{obs}} q_{\mathrm{obs}} \cos 2\left(P A_{\mathrm{obs}}-P A_{i}\right), \\
u_{\mathrm{corr}}=p_{\mathrm{obs}} u_{\mathrm{obs}} \cos 2\left(P A_{\mathrm{obs}}-P A_{i}\right),
\end{gathered}
$$

where $P A_{i}$ is the position angle of the instrumental polarization determined from the polarizance test.

The polarization and position angle measurements of the two high-polarization standards observed on each night were respectively found to be within $0.1 \%$ and $3^{\circ}$ of the references (Schmidt et al. 1992; Wolff et al. 1996). 
Table 1. Journal of spectropolarimetric observations.

\begin{tabular}{ccccccc}
\hline \hline $\begin{array}{c}\text { UT Date } \\
(\text { MM-DD-YYYY) }\end{array}$ & MJD & $\begin{array}{c}\text { Phase }^{a} \\
(\text { days })\end{array}$ & Airmass Range & $\begin{array}{c}\text { Avg. Seeing } \\
(\operatorname{arcsec})\end{array}$ & $\begin{array}{c}\text { Wavelength Range } \\
(\AA)\end{array}$ & $\begin{array}{c}\text { Exposure Time }^{b} \\
(\mathrm{~s})\end{array}$ \\
\hline $05-05-2019$ & 58607.3 & -10.9 & $1.04 \rightarrow 1.03$ & 1.30 & $4570-9000$ & $4 \times 1080$ \\
$05-06-2019$ & 58608.3 & -9.9 & $1.04 \rightarrow 1.13$ & 1.21 & $4570-9000$ & $4 \times 1080$ \\
$05-12-2019$ & 58614.3 & -3.9 & $1.01 \rightarrow 1.14$ & 1.63 & $4570-9000$ & $4 \times 1080$ \\
$05-13-2019$ & 58615.4 & -2.9 & $1.03 \rightarrow 1.13$ & 1.29 & $4570-9000$ & $4 \times 1080$ \\
$05-26-2019$ & 58628.4 & +10.1 & $1.09 \rightarrow 1.25$ & 1.86 & $4570-9000$ & $4 \times 1080$ \\
\hline \multicolumn{7}{c}{ Relative to $B$-band peak brightness at MJD 58618.2 (Kawabata et al. 2020). } \\
\\
\multicolumn{6}{c}{ Number of waveplate positions $\times$ exposure time at each position. }
\end{tabular}

\subsection{Interstellar Polarization}

Light passing through interstellar dust clouds is polarized through dichroic extinction by nonspherical paramagnetic dust grains present along the line of sight. The contribution to polarization by dust, namely the interstellar polarization (ISP), must be removed to determine the intrinsic polarization of the source. Although several approaches have been commonly used to estimate the ISP along the SN-Earth line of sight (see, for example, Stevance et al. 2019; Yang et al. 2020), the exact level of the ISP contribution to the observed polarization of SN 2019ein is generally uncertain.

Serkowski et al. (1975) showed that the Galactic ISP can be constrained to $p_{\text {ISP }}<9 \times E(B-V) \%$. The Milky Way colour excess $E(B-V)_{\text {MW }}$ along the line of sight of SN 2019ein is $0.011 \mathrm{mag}$ (Schlafly \& Finkbeiner 2011), constraining ISP $_{\text {MW }}$ to $<0.1 \%$. This upper limit is commensurate with the measured polarization of an ISP "probe star" - an intrinsically unpolarized star within $1^{\circ}$ of SN 2019ein that probes at least $150 \mathrm{pc}$ scale height of the Galactic interstellar medium (ISM). The Stokes $q$ and $u$ measured for the probe star were found to be $<0.05 \%$ in the continuum wavelength range of SN 2019ein, indicating low contribution from Galactic reddening.

An upper limit of the ISP from the host galaxy of SN 2019ein (NGC $5353)$ can be estimated based on the host reddening $E(B-V)_{\text {host }}=$ $0.09 \pm 0.02 \mathrm{mag}$ (Kawabata et al. 2020). Accounting for both the Galactic and SN2019ein-host dust, we place an upper limit of $p_{\text {ISP }}<0.9 \%$ along the SN-Earth line of sight. Such a value of ISP is higher than the continuum polarization level seen in SN 2019ein; thus, interstellar dust could potentially account for all of the continuum polarization of SN 2019ein. The caveat, however, is that Serkowski's law may not be applicable to all galaxies because different dust properties could lead to different efficiencies for ISP (Leonard et al. 2002). Therefore, we employ a more direct approach by following the method used by Yang et al. (2020) to estimate the ISP Stokes parameters $q_{\text {ISP }}$ and $u_{\text {ISP. }}$.

We consider the wavelength region $4800-5600 \AA$ in the spectrum when the $\mathrm{SN}$ is near its peak brightness. This region is expected to be intrinsically depolarized owing to multiple overlapping $\mathrm{Fe}$ absorption features, which create a "line blanketing" effect whose opacity dominates over electron scattering (Howell et al. 2001; Maund et al. 2013). We set the level of Stokes $q$ and $u$ to 0 within this wavelength range on day -2.9 (see Fig. 4), giving us an estimate of $q_{\text {ISP }} \approx-0.24 \%$ and $u_{\text {ISP }} \approx 0.19 \%$. We note that these estimates are consistent with the upper limit derived above using Serkowski's rule. These ISP values were subtracted from the observed $q$ and $u$ on each night and the polarization and the position angle were subsequently recalculated (see Table 2). We note that owing to the relatively low level of expected ISP, only a wavelength-independent ISP estimation is presented. We

4 We observed the star Gaia DR2 1497177392672672128.

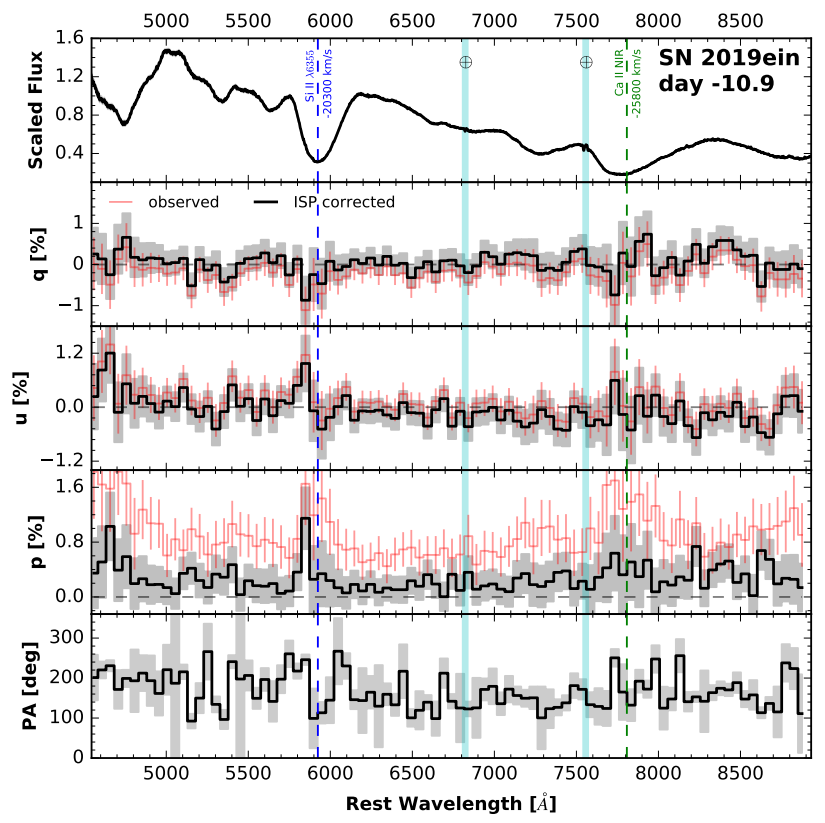

Figure 1. Spectropolarimetry of SN2019ein at day -10.9 relative to the $B$-band peak brightness at MJD 58618.2 (Kawabata et al. 2020). The cyan vertical bands represent the regions of telluric correction. The panels below the total-flux spectrum represent the polarimetry before (red) and after (black) the ISP correction. The grey-shaded area indicates the associated $1 \sigma$ uncertainty. The $P A$ panel shows only the polarization position angle after ISP correction. With the exception of the flux spectrum, we use $50 \AA$ binning for the purpose of presentation.

will discuss the ISP-corrected continuum and line polarization of SN 2019ein in Sections 3.4 and 3.5, respectively.

\subsection{Continuum Polarization}

Aspherical distribution of electrons, for instance an ellipsoidal photosphere, will cause imperfect cancellation of polarization E-vectors, leading to a nonzero continuum polarization (Höflich 1991, 1995; Bulla et al. 2015; Stevance et al. 2019). SNe Ia typically show low continuum polarization ( $\lesssim 0.3 \%$; see, for example, Wang \& Wheeler 2008; Yang et al. 2020), indicating that SNe Ia tend to be remarkably close to being spherical.

The continuum polarization of SN 2019ein and the associated uncertainty were estimated by binning the Stokes parameters over a wavelength range of $6400-7150 \AA$ following a procedure similar to 
Table 2. Summary of polarimetry results.

\begin{tabular}{|c|c|c|c|c|c|c|c|c|c|c|}
\hline MJD & $\begin{array}{c}\text { Phase }^{a} \\
\text { (d) }\end{array}$ & Instrument & $q(\%)$ & $\begin{array}{l}u(\%) \\
\quad \text { (obse }\end{array}$ & $\begin{array}{l}p(\%) \\
\text { ed) }\end{array}$ & $P A(\mathrm{deg})$ & $q(\%)$ & \multicolumn{3}{|c|}{ (ISP corrected) } \\
\hline 58607.3 & -10.9 & Kast & $-0.23(07)$ & $0.05(07)$ & $0.21(07)$ & $83(9)$ & $0.01(07)$ & $-0.14(07)$ & $\begin{array}{l}0.10(07) \\
\end{array}$ & $138(15)$ \\
\hline 58608.3 & -9.9 & Kast & $-0.18(05)$ & $0.09(05)$ & $0.19(05)$ & $77(7)$ & $0.06(05)$ & $-0.10(05)$ & $0.10(05)$ & 151(12) \\
\hline 58612.9 & -5.3 & RINGO3: $b$ & $-0.50(16)$ & $0.43(15)$ & $0.64(15)$ & $70(7)$ & $-0.13(25)$ & $-0.04(24)$ & $<0.01$ & $99(>360)$ \\
\hline 58614.3 & -3.9 & Kast & $-0.22(02)$ & $0.22(02)$ & $0.31(02)$ & $68(2)$ & $0.02(02)$ & $0.03(02)$ & $0.02(02)$ & $210(18)$ \\
\hline 58615.4 & -2.9 & Kast & $-0.23(04)$ & $0.23(04)$ & $0.32(04)$ & $68(3)$ & $0.01(04)$ & $0.03(04)$ & $0.00(04)$ & $215(30)$ \\
\hline 58620.0 & 1.8 & RINGO3: $b$ & $-0.33(15)$ & $0.64(16)$ & $0.70(15)$ & $59(6)$ & $0.04(24)$ & $0.17(25)$ & $<0.01$ & $219(>360)$ \\
\hline 58622.9 & 4.7 & RINGO3: $b$ & $-0.28(15)$ & $0.34(13)$ & $0.42(12)$ & $65(9)$ & $0.09(24)$ & $-0.13(22)$ & $<0.01$ & $152(>360)$ \\
\hline 58628.4 & 10.1 & Kast & $-0.07(06)$ & $-0.05(07)$ & $0.03(07)$ & $108(24)$ & $0.17(06)$ & $-0.24(07)$ & $0.28(07)$ & $153(7)$ \\
\hline 58630.9 & 12.7 & RINGO3: $b$ & $-0.15(24)$ & $1.14(26)$ & $1.12(25)$ & $49(6)$ & $0.22(33)$ & $0.67(35)$ & $0.54(34)$ & $215(17)$ \\
\hline 58639.0 & 20.8 & RINGO3: $b$ & $-0.47(23)$ & $-0.91(23)$ & $0.99(22)$ & $122(7)$ & $-0.10(32)$ & $-1.38(32)$ & $1.31(32)$ & $133(6)$ \\
\hline
\end{tabular}

${ }^{a}$ Relative to $B$-band peak brightness at MJD 58618.2 (Kawabata et al. 2020).

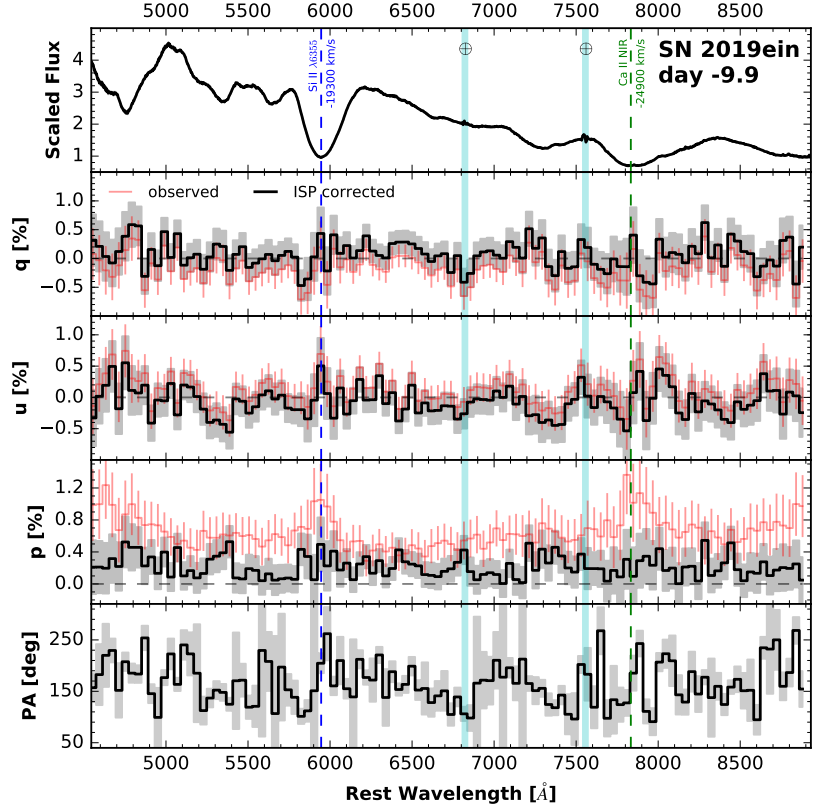

Figure 2. Similar to Figure 1 but for day -9.9 . We use $40 \AA$ binning for the purpose of presentation.

that described by Yang et al. (2020). The selected spectral region is known to be free from strong absorption features (Patat et al. 2009). The uncertainty was correspondingly binned.

The continuum polarization on days -10.9 and -9.9 is low with values of $p_{\text {cont, }-10.9 \mathrm{~d}}=0.10 \pm 0.07 \%$ and $p_{\text {cont, }-9.9 \mathrm{~d}}=0.10 \pm 0.05 \%$, respectively (Figs. 1 and 2). The polarization is consistent with 0 as the $\mathrm{SN}$ approaches maximum brightness around day -3.9 and day -2.9 (Figs. 3 and 4). After peak brightness, polarization increases slightly, reaching $p_{\text {cont },+10.1 \mathrm{~d}}=0.28 \pm 0.07 \%$ (Fig. 5). We note that these values are consistent with infrared spectropolarimetry of SN 2019ein, which found a $3 \sigma$ upper limit on polarization of $1.2 \%$ around the SN peak brightness (Tinyanont et al. 2021).

The measured continuum polarization position angle $(P A)$, which represents the position of global axisymmetry of the ejecta, remains fairly consistent before and after peak brightness, hovering around $150^{\circ}$. Even though the $P A$ on days -3.9 and -2.9 is apparently larger

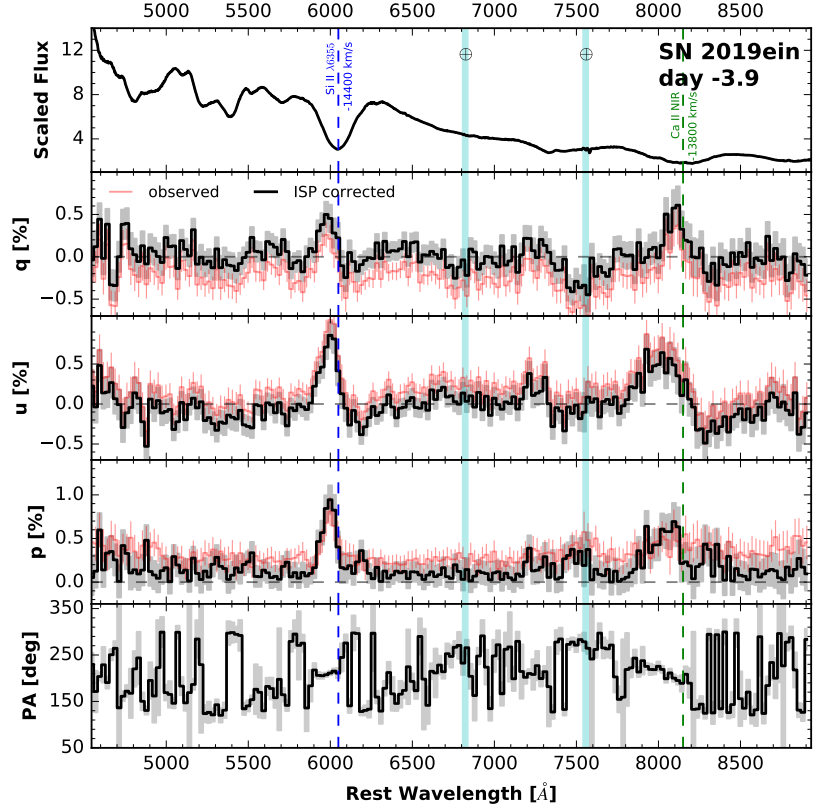

Figure 3. Similar to Fig. 1 but for day -3.9 . We use $25 \AA$ binning for the purpose of presentation.

$\left(\sim 210^{\circ}\right)$, we note that the polarization is so close to zero that the $P A$ is essentially undetermined on those days.

\subsection{Line Polarization}

Polarization signal at specific spectral lines arises due to the presence of clumps of material above the photosphere. The absorbing material partially obscures the underlying Thomson-scattering photosphere, resulting in an excess of polarization superimposed on the continuum polarization over the range of the absorption wavelengths. Below, we present the polarization of three absorption features as follows.

(i) Si i 16355 : We observe weak line polarization $(0.5 \pm 0.4 \%)$ on day -10.9 , whereas no significant line polarization was detected on day -9.9 . On days -3.9 and -2.9 , we see strong line polarization, which reaches its peak value of $\sim 1 \%$ at an expansion velocity of 


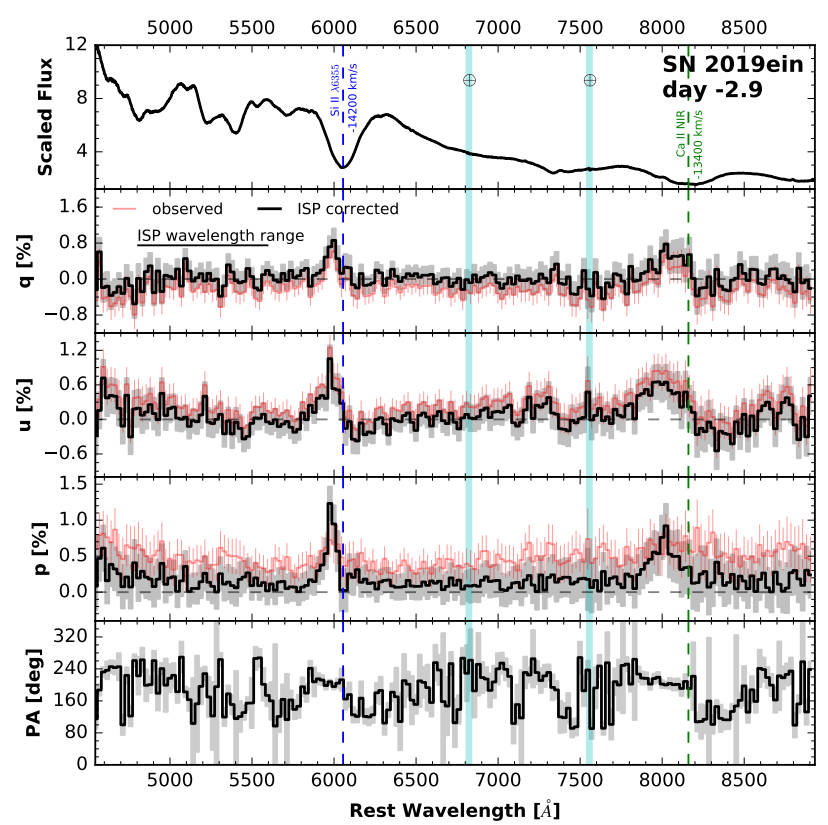

Figure 4. Similar to Fig. 1 but for day -2.9 . We use $25 \AA$ binning for the purpose of presentation. The wavelength region $4800-5600 \AA$ was used to estimate the ISP as described in Section 3.3.

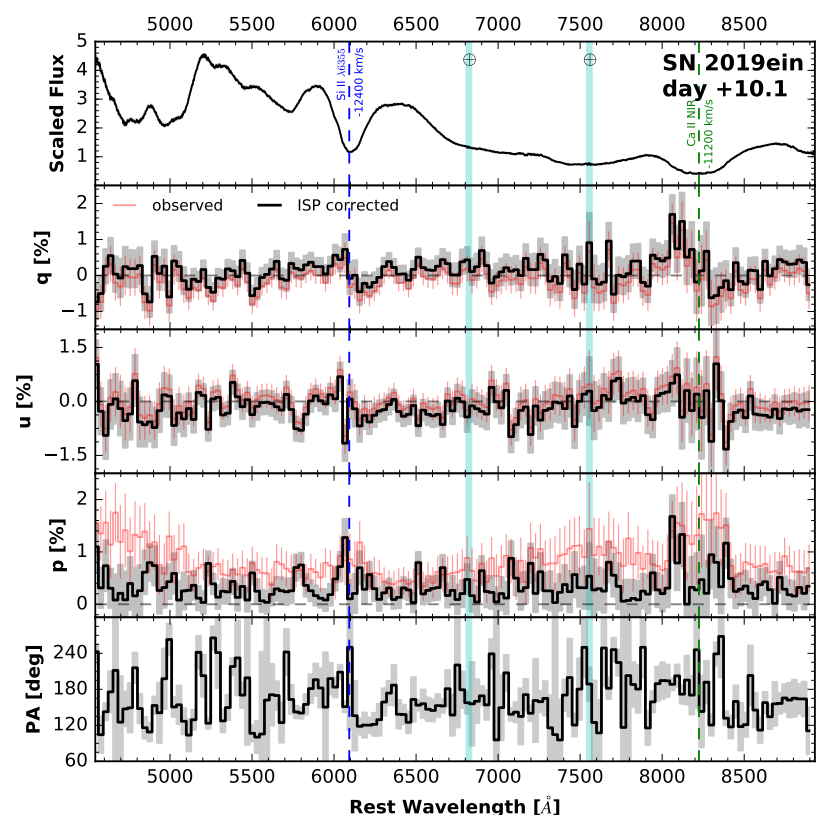

Figure 5. Similar to Fig. 1 but for day +10.1 . We use $30 \AA$ binning for the purpose of presentation. $\sim 17,000 \mathrm{~km} \mathrm{~s}^{-1}$. The strong line polarization persists into day 10.1, at an expansion velocity of $\sim 13,600 \mathrm{~km} \mathrm{~s}^{-1}$.

(ii) О г $\lambda 7774$ : No significant line polarization was seen at any phase.

(iii) Ca II NIR3: Similarly to Si II, no significant line polarization was observed on days -10.9 and -9.9 . In contrast, on days -3.9 and -2.9 , we see strong line polarization, reaching its peak value of $\sim 0.8 \%$ at an expansion velocity of $\sim 18,000 \mathrm{~km} \mathrm{~s}^{-1}$. The strong line polarization continues into day 10.1 , reaching up to $\sim 1.5 \%$ at $17,000 \mathrm{~km} \mathrm{~s}^{-1}$.

Starting on day -3.9 and thereafter, both Si II and Ca II show a complex structure in the polarization spectra, likely associated with high-velocity (HV) and normal-velocity (NV) components. For example, at day +10.1 , the polarization across $\mathrm{Ca}$ II NIR3 reached two local maxima at $-17,000 \mathrm{~km} \mathrm{~s}^{-1}$ and $-5900 \mathrm{~km} \mathrm{~s}^{-1}$. The emergence of the line polarization of both the $\mathrm{HV}$ and $\mathrm{NV}$ components over time may result from an increase of $\mathrm{Si}$ II and $\mathrm{Ca}$ II opacity from larger to smaller radii.

\subsection{The $q-u$ Plane and the Dominant Axis}

Plotting the Stokes parameters in the $q-u$ plane allows us to examine the axisymmetry of the continuum and various spectral features. If the $\mathrm{SN}$ ejecta are smooth and axisymmetric, the data points should fall along a straight line called the "dominant axis" (Wang et al. 2001, 2003). Deviations from the dominant axis in the perpendicular direction represent departures from axisymmetry and clumpiness of the ejecta. The dominant axis is determined by

$u=\alpha q+\beta$,

where $\alpha$ and $\beta$ are the fitted parameters from an error-weighted orthogonal distance regression. In Figure 6, we present the polarization in the $q-u$ plane in the continuum as well as for Si II $\lambda 6355$ and $\mathrm{Ca}$ II NIR3. We omitted the Si II and Ca II lines when plotting the continuum $q$ and $u$ in the wavelength range 4700-8750 $\AA$. The fitted parameters $\alpha$ and $\beta$ that characterise the dominant axes are given in Table 3.

As suggested by the values of $\chi^{2} / D o F$ in Figure 6, the departure from the dominant axis fitted across the line profile indicates a significant clumpiness in the Si II-rich ejecta on days -10.9 and -9.9 . Considering the relatively large values of $\chi^{2} / D o F$, it is ambiguous whether a dominant axis is present at early times. The absence of a clear dominant axis together with the measured low polarization suggests that any $\mathrm{Si}$ II-rich clumps are fairly uniformly distributed in the ejecta at early times, when the photosphere only intersects with the outermost part of the ejecta. Axial symmetry is evident on days -3.9 and -2.9 for $\mathrm{Si}$ II when the SN is near its peak brightness. However, the axial symmetry becomes weak and clumpiness increases again around day +10 . Ca II shows an overall higher degree of clumpiness compared to $\mathrm{Si}$ II. Ca II also exhibits weak axial symmetry at early times. However, as time progresses, Ca II settles onto a more prominent symmetry axis. Unlike Si II, Ca II continues to exhibit high axial symmetry at day +10 . The symmetry axes of both $\mathrm{Si}$ II and $\mathrm{Ca}$ II are roughly aligned with each other and remain fairly constant starting on day -10 and thereafter.

As seen in the left panels of Figure 6, no clear dominant axis can be identified in the continuum at any epoch. The data points form a cloud centred near the origin. This strengthens the case that even though the overall ejecta geometry is spherical, the polarization in $\mathrm{Si}$ II and $\mathrm{Ca}$ II is due to clumps of explosively synthesised material. 

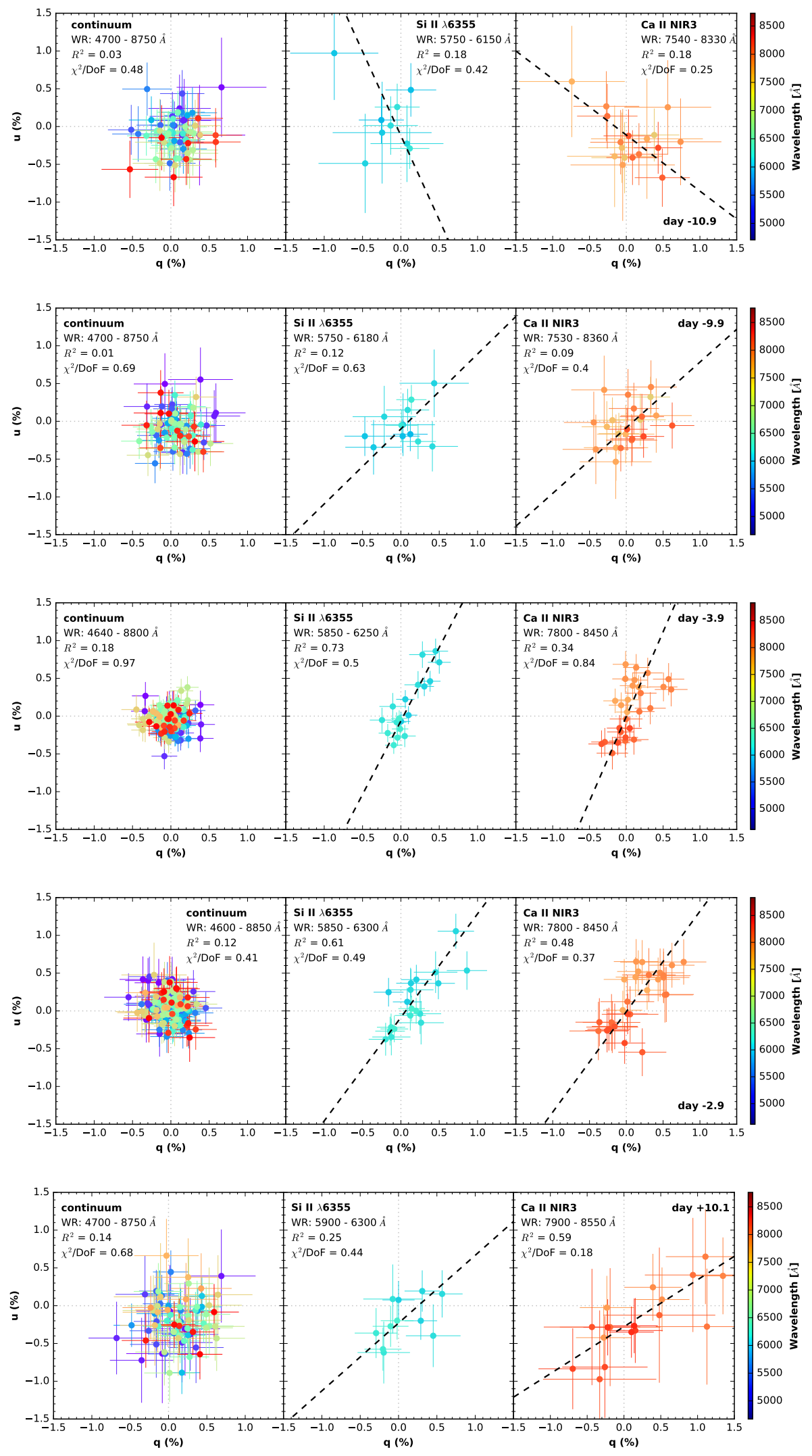

Figure 6. Polarization in the Stokes $q-u$ plane. The Si II $\lambda 6355$ and Ca II NIR3 lines were omited from the left-side panels labeled "continuum". The dashed lines in the "Si II 26355 " and the "Ca II NIR3" panels represent the dominant axes for the labeled absorption features. 
Table 3. Fitted parameters for the dominant axes.

\begin{tabular}{c|cc|ccc|ccc}
\hline \hline $\begin{array}{l}\text { Phase } \\
(\text { days })\end{array}$ & $\alpha_{\text {cont }}$ & $\beta_{\text {cont }}$ & $\alpha_{\text {Si II }}$ & $\beta_{\text {Si II }}$ & $\begin{array}{c}\text { Velocity Range } \\
\left(-10^{3} \mathrm{~km} \mathrm{~s}^{-1}\right)\end{array}$ & $\alpha_{\text {Ca II NIR3 }}$ & $\beta_{\text {Ca II NIR3 }}$ & $\begin{array}{c}\text { Velocity Range } \\
\left(-10^{3} \mathrm{~km} \mathrm{~s}^{-1}\right)\end{array}$ \\
\hline-10.9 & $0.81(21)$ & $-0.15(04)$ & $-2.23(1.35)$ & $-0.12(23)$ & $28.6-9.7$ & $-0.74(24)$ & $-0.11(07)$ & $34.8-6.7$ \\
-9.9 & $4.68(2.62)$ & $-0.47(24)$ & $0.99(49)$ & $-0.10(09)$ & $28.5-7.3$ & $0.87(28)$ & $-0.08(06)$ & $34.8-6.7$ \\
-3.9 & $0.33(09)$ & $-0.04(01)$ & $1.95(27)$ & $-0.06(05)$ & $23.9-5.0$ & $2.24(52)$ & $0.00(08)$ & $26.1-3.2$ \\
-2.9 & $-0.94(14)$ & $0.03(02)$ & $1.37(24)$ & $-0.09(06)$ & $23.8-3.9$ & $1.33(23)$ & $-0.01(07)$ & $26.1-3.2$ \\
+10.1 & $0.02(13)$ & $-0.20(04)$ & $0.90(31)$ & $-0.23(08)$ & $21.5-2.6$ & $0.62(11)$ & $-0.28(06)$ & $22.5-0.3$ \\
\hline
\end{tabular}

\subsection{Polarization Time Series}

We build a temporal series of polarization measurements of SN2019ein by combining the Kast spectropolarimetry and the RINGO3 imaging polarimetry. In order to compare the polarization measured by the two instruments, we binned the Kast spectropolarimetry over the RINGO3 $b, g$, and $r$ filter passbands. This process estimates the equivalent imaging polarimetry data points in RINGO3 filters. In this way, we built the polarimetric dataset with a time baseline from -11 to 21 days relative to the $B$-band light-curve peak of SN 2019ein. The broad-band polarization from Kast was derived by integrating over the wavelength of the filter-transmission-weighted polarized flux.

The combined polarization time series is presented in Figure 7. The top panel displays two light curves obtained in the Landolt $I$ and $B$ bands with the Katzman Automatic Imaging Telescope (KAIT; Filippenko et al. 2001) at Lick Observatory. The middle panel shows polarization over time. The red, blue, and green circles represent the synthesised Kast polarization in wavelength ranges that roughly match the three channels of RINGO3. The black squares present the Kast continuum polarization in the wavelength range 6400-7150 A. We note that the $b$ and $r$ bins include the polarization of the Si II $\lambda 6355$ and Ca II NIR3 features, respectively. The blue inverted triangles show the polarization measured by the blue channel of RINGO3. The bottom panel provides the measured $P A$ in the continuum region (6400-7150 $\AA$ ) by Kast and by the blue channel of RINGO3. The polarimetry presented here has been ISP corrected. Since we do not know the exact magnitude of any systematic bias (for example, instrumental polarization) in RINGO3 measurements, we employed a different strategy to account for ISP and any systematic bias: we calculated the mean $q$ and $u$ from the three epochs within $\sim 5$ days of the $B$-band peak brightness (days $-5.3,+1.8$, and +4.7 ; see Table 2 ), which gives $q_{\mathrm{ISP}+\mathrm{sys}} \approx-0.37 \%$ and $u_{\mathrm{ISP}+\mathrm{sys}} \approx 0.47 \%$. We then subtracted the averaged $q$ and $u$ from the observed Stokes parameters of all RINGO3 data under the assumption that SNe Ia exhibit effectively zero continuum polarization near peak brightness. This assumption is validated independently by the Kast spectropolarimetry of SN 2019ein on days -2.9 and -3.9 . We also propagated the uncertainty of ISP subtraction into the final calculations of $p$ and $P A$.

\section{DISCUSSION}

A nonzero continuum polarization may result from either an overall inhomogeneous electron density distribution or a nonspherical heating source. The latter case was seen in models of Bulla et al. (2016a,b) for SNe Ia, and has also been used to explain the observed increase in polarization during the plateau phase of SNe IIP, in which an aspherical ionisation front of ${ }^{56} \mathrm{Ni}$ is typically present (Höflich et al. 1996).

Overall, the polarization properties of SN 2019ein before its peak luminosity are consistent with the typical behaviour of SNe Ia. For example, the continuum polarization is $<0.2 \%$. Distinct line polarization, which is typically on the order of $1 \%$, can also be identified across some prominent spectral lines including Si II, Fe II, and Ca II (Wang \& Wheeler 2008). The asymmetric distribution of the intermediate-mass elements (IMEs, $9 \leqslant Z \leqslant 20$, including $\mathrm{Si}, \mathrm{Ca}, \mathrm{S}$, and $\mathrm{Mg}$ ) inferred from the line polarization is indicative of sufficient outward mixing. IMEs generated in the nuclear burning can also be produced at higher velocities compared to the SN ejecta. In thermonuclear $\mathrm{SNe}$, below the production zone of the IMEs, the inner burning region is surrounded by $\mathrm{C}$ and $\mathrm{O}$ from the progenitor WD. The energy input is given by the radioactive decay chain of ${ }^{56} \mathrm{Ni} \rightarrow{ }^{56} \mathrm{Co} \rightarrow{ }^{56} \mathrm{Fe}$, which is initiated by the nucleosynthesis of ${ }^{56} \mathrm{Ni}$ as the main product of the silicon-burning process. An asymmetric ${ }^{56} \mathrm{Ni}$ distribution hence results in an asymmetric energy source.

The low line and continuum polarization at early times (day -11) do not support the idea put forward by Pellegrino et al. (2020) that the bluesfhited emission peaks in early-time spectra are due to an aspherical explosion enhancing abundance of material at high velocities. Furthermore, the lack of a clear dominant axis in the $q-u$ plane at early times indicates that even if clumps of high-velocity material are present, they must be fairly uniformly distributed in the outer ejecta. We cannot, however, rule out the possibility that the apparent blueshift is due to optical-depth effects arising from a steep density profile in the ejecta (Pellegrino et al. 2020).

As seen in the bottom panel of Figure 7, we do not identify significant variation in $P A$ at different epochs. We note that near peak brightness, $q$ and $u$ intrinsic to SN 2019ein are very close to zero after correcting for the ISP as discussed in Section 3.7. Such small values of Stokes parameters lead to effectively random values of the $P A$ around the SN light-curve peak. Furthermore, we remark that the $P A$ calculated before ISP removal also tends to be consistent from day -11 to +21 (see Table 2 ). A relatively low level of continuum polarization together with a roughly constant direction of the dominant axis suggests a common axial symmetry from the outer electron-scattering zone to the inner region near the energy source.

We remark that RINGO3 uses two dichroics to separate the three wavelength channels and a depolarizing Lyot prism, resulting in an induced systematic uncertainty in polarization of up to $\sim 0.5 \%$ (Jermak 2017). As shown in the third and the fourth panels of Figure 7 , after day +5 , the continuum level of polarization estimated from RINGO3 observations is mostly from $u$, while $q$ is consistent with zero. A moderate degree of asphericity is suggested by Kast spectropolarimetry at day +10 . Unfortunately, we were not able to conduct Kast spectropolarimetry of SN 2019ein after day +10 owing to technical issues. Therefore, the "rise" in polarization after maximum brightness is anchored by just one RINGO3 measurement from day +21 . For this reason, out of an abundance of caution, we refrain from claiming that a definitive rise in polarization was observed in SN 2019ein post maximum brightness. However, if the post-peak rise of polarization is real and intrinsic to SN 2019ein, we cautiously provide our interpretations below, hoping to invite more sophisticated theoretical investigations. 


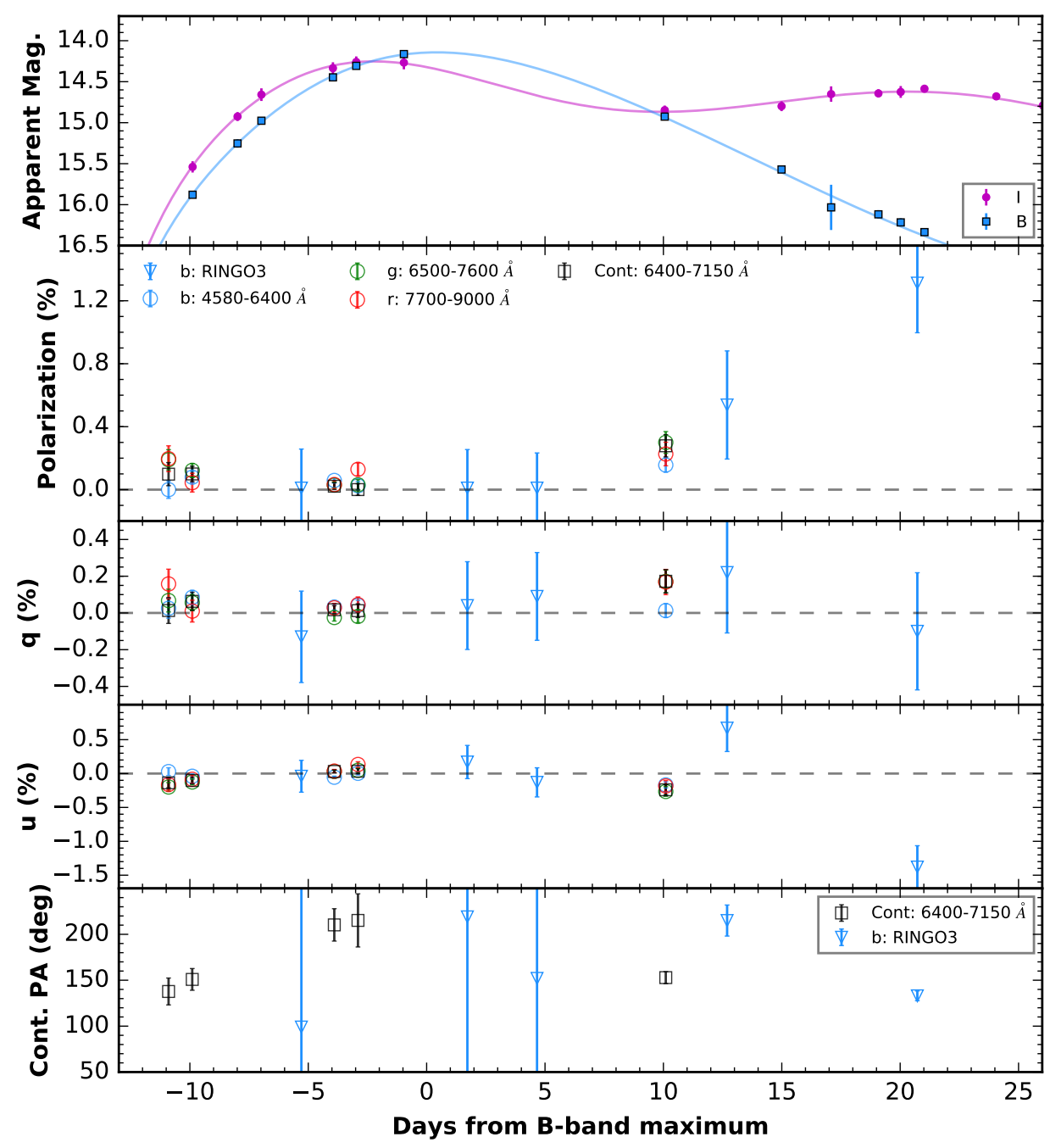

Figure 7. Polarization vs. time. The polarization and $P A$ have been corrected for the ISP. The black squares represent the continuum polarization and $P A$ from the Kast spectropolarimeter. The red, blue, and green circles show the polarization measured by Kast in different wavelength regions: blue (4580-6400 $\AA$ ), green (6500-7600 $)$, and red (7700-9000 ̊). The blue inverted triangles show polarization and PA measured by RINGO3 in the blue channel with wavelength range $3500-6400 \AA$. The light curves in the top panel were obtained by KAIT at Lick Observatory.

We suggest that the secondary maximum in the NIR luminosity could be the key for understanding the post-peak rise of the continuum polarization. The formation of the secondary maximum in the NIR can be understood as an opacity effect. To first order, the NIR luminosity is given by $L_{\mathrm{NIR}}(t) \propto T(t)^{4} \times R(t)^{2}$, where $T(t)$ and $R(t)$ represent the temperature and the radius of the photosphere as a function of time, respectively. In normal SNe Ia, although the photospheric radius increases with time because the opacity remains high for 2-3 weeks after the SN explosion, it recedes gradually in mass coordinate (see, e.g., Fig. 3 of Höflich 2017) until the SN light curve approaches its secondary maximum in the NIR. This may explain the relatively little evolution of polarization seen in SN 2019ein until peak brightness.
After peak brightness, the recession of the SN photosphere is governed by the geometrical dilution of the homologously expanding envelope. However, $R(t)$ decreases rapidly owing to the significant drop in opacity when Fe-group elements begin recombining from ionisation states III to II (Höflich et al. 1993, 1998; Kasen et al. 2006). Therefore, when the SN reaches its secondary NIR maximum $\sim 40-50$ days after the explosion, most of the energy input emerges above the photosphere. In the presence of an asymmetric energy source, the flux at the photosphere will be direction-dependent. Consequently, an inhomogeneous photosphere could develop, leading to rise in polarization of the SN after maximum brightness. The interpretation of any rise in late-time polarization may be complicated by optical-depth effects that still remain poorly understood (Höflich 
1991) in SNe photospheres. As such, detailed theoretical investigations into how polarization behaves over time for various explosion mechanisms are pressingly warranted.

On the other hand, if the rise in late-time polarization is shown to be not true and the inner energy source is found to be spherical, we also arrive at an interesting implication. In that case, models with burning starting on the surface might be better at explaining the observed polarimetry. We speculate that if the burning starts on the surface, the detonation will propagate through the central zone supersonically and preserve the spherical nature of the WD.

Spectropolarimetric observations of SNe Ia beyond 20 days after maximum light are very rare. The handful of SNe Ia for which such measurements exist display low continuum polarization. For example, SN $2012 \mathrm{fr}$ was polarized to $\sim 0.2 \%$ on day +24 (Maund et al. 2013), and SN 2001el and SN 2006X showed $<0.1 \%$ polarization on days +38 and +39 , respectively (Patat et al. 2009; Wang et al. 2003). These observations challenge the apparent late-time increase in polarization of SN2019ein. However, owing to a small sample and the fact that these SNe display a diverse set of properties (expansion velocities, decline rates, etc.), the question remains whether any $\mathrm{SNe}$ Ia display late-time increases in polarization. More polarimetric observations of SNe Ia at similar phases are required to answer that question.

\subsection{Implications for Explosion Scenarios}

The time-invariant $P A$ observed from early times to the phase just prior to the secondary maximum in the NIR light curves of SN 2019ein brings us to the implications for explosion scenarios and the characteristics of $p$. Based on current understanding, SNe Ia might be triggered through the following mechanisms.

(i) Deflagration: compressional heating in a slow accretion near the WD centre triggers subsonic burning when the WD approaches the Chandrasekhar mass $M_{\mathrm{Ch}}$ (Nomoto et al. 1984; Gamezo et al. 2004; Röpke 2007; Ma et al. 2014).

(ii) Delayed detonation: explosion begins at the end of a deflagration phase near or at the centre of the WD. When a critical density of $\sim 10^{7} \mathrm{~g} \mathrm{~cm}^{-3}$ is reached a transition to detonation occurs (Khokhlov 1991).

(iii) Colliding/inspiraling WDs: heat released on dynamical timescales triggers a detonation of a double-degenerate system (Iben \& Tutukov 1984; Webbink 1984; Benz et al. 1990; Nugent et al. 1997; Pakmor et al. 2010; Kushnir et al. 2013; García-Berro \& LorénAguilar 2017).

(iv) Double/helium detonation: a sub- $M_{\mathrm{Ch}} \mathrm{C}-\mathrm{O}$ WD may explode by detonating a thin surface He layer, which triggers a detonation front in the WD (Woosley et al. 1980; Nomoto 1982a,b; Livne 1990; Woosley \& Weaver 1994; Höflich \& Khokhlov 1996; Kromer et al. 2010).

In the framework of off-centre delayed-detonation models, a slightly asymmetric excitation will lead to an off-centre distribution of iron-group elements. The axis of symmetry will be defined by the centroid of the density distribution and the point of off-centre delayed-detonation transition (Livne 1999; Höflich et al. 2006; Fesen et al. 2007). If the ${ }^{56} \mathrm{Ni}$ region is above the photosphere, the time-invariant $P A$ observed in SN 2019ein would indicate a moderate asphericity of the central energy source. Otherwise, a change of $P A$ may be seen as the SN approaches its secondary peak. The low continuum polarization observed in SN2019ein challenges any model that predicts significant asymmetry of the photosphere.

Dynamical or head-on collisions of WDs are expected to show larger asymmetries (Benz et al. 1990; Pakmor et al. 2011; Sato et al. 2016; Katz et al. 2016; García-Berro et al. 2017; García-Berro \& Lorén-Aguilar 2017). The dynamical models are not favoured since they predict high polarization levels at early phases (Pakmor et al. 2012; Bulla et al. 2016a) and larger asymmetries in the inner layers or off-centred energy sources, which are incompatible with our observations of SN 2019ein.

For sub- $M_{\mathrm{Ch}}$ explosions through a double/helium detonation, outer asymmetry is expected owing to the He-ignition process. Classical He-detonation models require a significant He-surface mass on the order of 0.01 to $0.1 M_{\odot}$ (e.g., Nomoto 1982a,b; Woosley \& Weaver 1994; Höflich \& Khokhlov 1996; Bildsten et al. 2007; Shen $\&$ Bildsten 2009). Starting from the SN explosion, the photosphere recedes and will first cross the burning product of the outermost He layer. The O I $\lambda 7774$ feature is prominent at day -10.9 in the flux spectrum. However, we see no polarization signal at the corresponding wavelength, suggesting that oxygen was present in the outer layer and maintained a spherically symmetric distribution in the expanding envelope. This is in contrast with the $\sim 0.4 \% \mathrm{O}_{\mathrm{I}}$ $\lambda 7774$ line polarization predicted by Bulla et al. (2016b) for the double/helium detonation models. Therefore, we infer that the outermost layer is dominated by the spherical pre-explosion $\mathrm{C}$ and $\mathrm{O}$ from the $\mathrm{WD}$, rather than a He shell, since the latter is likely to produce an abundance jump in $\mathrm{O}$ in the line-forming region (see, e.g., Yang et al. 2020). In fact, SNe 2006X and 2004dt, both of which are highvelocity $\mathrm{SNe}$ Ia, also show no polarization across $\mathrm{O}$ I, putting strong constraints on the distribution of oxygen in the explosion ejecta. We note that the polarimetric properties of more modern models of sub$M_{\text {Ch }}$ double/helium detonation (e.g., Shen et al. 2018) are currently theoretically unexamined.

\subsection{Comparison with a Detonating Failed Deflagration (DFD) Model}

Kasen \& Plewa (2007) made theoretical predictions for spectropolarimetric observations of a $M_{\mathrm{Ch}}$ WD using the detonating failed deflagration (DFD) model. They studied one particular model in detail, named Y12, in which the WD's ignition starts within a small spherical region, $50 \mathrm{~km}$ in diameter and offset $12.5 \mathrm{~km}$ from the centre. Here, we compare the observed spectropolarimetric properties of SN 2019ein with the predictions of Kasen \& Plewa (2007).

According to Kasen \& Plewa (2007), if an SN is observed from the deflagration side - the side where ignition began - high ejecta velocities are expected. Since SN 2019ein exhibited very high expansion velocities, we may be observing the explosion from the ignition side (viewing angles $\theta \approx 0^{\circ}$ ). From this orientation, the projected surface of the intrinsically "egg-shaped" density structure in the observer's direction would be fairly circular, leading to low continuum polarization. In fact, Kasen \& Plewa (2007) argue that low continuum polarization is expected from all viewing angles at peak brightness. Therefore, continuum polarization is not informative for constraining the viewing angle of the SN.

The Y12 model predicts substantial line polarization (1-2\%) depending on the viewing angle (Fig. 13 of Kasen \& Plewa 2007). Indeed, we observe significant line polarization across both Si II $\lambda 6355$ and $\mathrm{Ca}$ II NIR3 features in SN2019ein, which suggests a viewing angle of $\theta \approx 0^{\circ}$ or $90^{\circ}$. A viewing angle of $\sim 180^{\circ}$ (opposite to the ignition side) is disfavoured because we observe high polarization across both $\mathrm{Si}$ II and $\mathrm{Ca}$ II, whereas in the Y12 model only Si II polarization is seen for angles $\sim 180^{\circ}$. Together with the high expansion velocity of SN 2019ein, $\theta \approx 0^{\circ}$ is favoured over other orientations, strengthening the case that we may be viewing the SN from the igni- 
tion side. According to Kasen \& Plewa (2007), such events are rare and expected to constitute roughly $10 \%$ of all SNe Ia. Spectropolarimetry of more SNe Ia is needed to test this prediction.

As described in Section 3.6, both $\mathrm{Si}$ II and Ca II display higher degree of clumpiness at early epochs than near and after peak brightness. This is also expected in the DFD model, which can produce a clumpy outer layer of IMEs but maintain a relatively smoother IME distribution in the inner layers.

\subsection{The Si II $\lambda 6355$ Polarization Compared with a Larger Sample}

We notice that the continuum polarization of SN2019ein on the Stokes $q-u$ diagram can be fitted with straight lines (e.g., see the left panels of Fig. 6). A dominant axis is present in Kast spectropolarimetry between days $\sim-11$ and +10 . Except for the first epoch, the direction of the dominant axis appears to be unchanged, suggesting that different layers of the ejecta share a roughly fixed axial symmetry. These properties indicate that SN 2019ein belongs to the spectropolarimetric type D1 (Wang \& Wheeler 2008), in which a dominant axis is identifiable but with significant scatter.

We compared the polarimetric properties of SN 2019ein with those of SNe 2004dt and 2006X, both of which display high expansion velocities at early phases. As inferred from the absorption minimum of the Si II $\lambda 6355$ line, SN 2004dt shows an expansion velocity of $\sim 17,000 \mathrm{~km} \mathrm{~s}^{-1}$ at $\sim 6-8$ days before the optical maximum (Wang et al. 2006), and SN 2006X exhibits an expansion velocity of $20,700 \mathrm{~km} \mathrm{~s}^{-1}$ at day -11.3 (Wang et al. 2008). A linear correlation between the maximum polarization measured across Si II $\lambda 6355$, $p_{\mathrm{SiII}}^{\max 5}$ and the $\mathrm{SN}$ expansion velocity traced by the same line at day $-5, v_{\text {SiII@-5d }}$, has been found by Cikota et al. (2019) based on the analysis of a sample of $35 \mathrm{SNe}$ Ia. The velocity-polarization relation connects the kinematics with the ejecta asymmetry and indicates that a higher departure from spherical symmetry for $\mathrm{Si}$ is produced at higher velocities.

For comparison with the Si II velocity-polarization relation presented in Figure 13 of Cikota et al. (2019), we estimated $v_{\text {SiII } @-5 d}=$ $15,100 \pm 300 \mathrm{~km} \mathrm{~s}^{-1}$ for SN 2019ein. The peak polarization of SN 2019ein across the Si II $\lambda 6355$ line, i.e., $p_{\text {SiII }}^{\max }$ derived based on $100 \AA$ and $50 \AA$ binnings on day -4 , is $0.76 \pm 0.10 \%$ and $0.82 \pm 0.16 \%$, respectively. These values place SN 2019ein slightly above the predicted Si II $\lambda 6355$ polarization. We remark that SN 2019ein is still broadly consistent with the Si II velocity-polarization relation, corroborating the trend that higher-velocity SNe Ia tend to exhibit higher polarization. SN 2019ein shows significantly lower Si II $\lambda 6355$ polarization compared to SN $2004 \mathrm{dt}\left(14,870 \pm 140 \mathrm{~km} \mathrm{~s}^{-1}, 1.34 \pm 0.14 \%\right)$, which exhibits an exceptionally high peak polarization across the Si II line and was considered an outlier by Cikota et al. (2019). On the other hand, SN 2006X $\left(17,040 \pm 90 \mathrm{~km} \mathrm{~s}^{-1}, 0.63 \pm 0.05 \%\right)$ is in good agreement with the $\mathrm{Si}$ II velocity-polarization relation.

Wang et al. (2007) also derived a correlation between the maximum line polarization of Si II $\lambda 6355$ and $\Delta m_{15}(B)$. For the former parameter, the observations are often converted to the level at five days before the $B$-band maximum, i.e., $p_{\text {SiII }}^{\text {corr }-5}$. Owing to the sparsely sampled spectropolarimetry, we adopt the peak Si II $\lambda 6355$ polarization measured at day -4 for SN2019ein. The $B$ band light-curve decline rate of SN 2019ein has been determined as $\Delta m_{15}(B)=1.36 \pm 0.02$ mag (Kawabata et al. 2020) and $\Delta m_{15}(B)=$

\footnotetext{
5 The peak of the Si II $\lambda 6355$ polarization is measured between roughly days -11 and +1 (Cikota et al. 2019)
}

$1.40 \pm 0.004$ mag (Pellegrino et al. 2020). We infer that SN 2019ein is consistent with the $\Delta m_{15}(B)-p_{\text {SiII }}^{\text {corr }}-5$ relation as presented by Wang et al. (2007) and Cikota et al. (2019). This relation can be interpreted such that at a given epoch, higher line polarization is expected for less-luminous $\mathrm{SNe}$, which indicates a higher chemical nonuniformity. This can be understood as an indication that less material is burned in fainter events, and such incomplete burning may not be sufficient to wipe out lumpy chemical configurations.

Therefore, we conclude that SN 2019ein is consistent with both the Si II velocity-polarization relationship and the light-curve decline rate-Si II polarization relation. Such behaviour may be explained with the off-centre delayed-detonation model (e.g., Höflich et al. 2006; Cikota et al. 2019).

\section{CONCLUDING SUMMARY}

We have presented spectropolarimetry of SN 2019ein, a high-velocity SN Ia in NGC 5353. Our observations range from day -10.9 to +10.1 from the $B$-band light-curve peak. We found that the continuum polarization in SN 2019ein is low, staying $<0.25 \%$ until about a month after the explosion. This indicates that the photosphere is quite close to being spherical.

The blueshifted emission peaks observed in early-time spectra of SN2019ein cannot be due to a highly asymmetric explosion, as evidenced by low continuum polarization at early times. However, our observations do not preclude the possibility that optical-depth effects in a steep-density ejecta lead to the apparent blueshift of the emission peaks.

The RINGO3 imaging polarimetry shows an apparent increase in polarization $(\sim 1 \%)$ around day +21 . However, owing to significant systematic uncertainties found in previous RINGO3 measurements, we are cautious of the observed rise in polarization. If the postpeak increase in polarization is real and intrinsic to SN 2019ein, we note that it coincides with the beginning of the transition from $\mathrm{Fe}$ III to Fe II ionisation states. The recombination decreases the opacity, providing us a deeper view into the $\mathrm{SN}$ ejecta. We speculate that the possible post-peak rise of the polarization, therefore, could indicate the presence of an aspherical central energy source.

The polarization position angle does not change drastically over the observed epochs. We also observe high line polarization $(\sim 1 \%)$ across the Si II $\lambda 6355$ and the Ca II NIR3 features around peak brightness of SN 2019ein. The polarization signatures of SN2019ein are consistent with models predicting SNe Ia explosions that produce a modest amount of asphericity. To summarise,

(i) A low amount of asphericity in the high-velocity layers is detected, as in previous observations of other SNe Ia.

(ii) Significant departures from global spherical symmetry can be ruled out throughout the ejecta. A common symmetry axis persists from the outer to the inner layers.

(iii) After day +21 , the possibility of a small amount of polarization caused by an asymmetric distribution of ${ }^{56} \mathrm{Ni}$, which may arise from many different models of SN explosions (Höflich 1991; Leonard et al. 2005; Kasen et al. 2009; Pakmor et al. 2010; Seitenzahl et al. 2013; Moll et al. 2014; Raskin et al. 2014; Bulla et al. 2015, 2016a,b; Höflich et al. 2017), cannot be eliminated. Spectropolarimetry of more $\mathrm{SNe}$ Ia at post-peak epochs is needed to confirm whether polarization rises beyond day +20 .

The spectropolarimetric observations of SN2019ein strengthen existing evidence that the explosions of SNe Ia are largely spherical, 
especially when considering that SN2019ein is an event with one of the highest expansion velocities ever observed.

Finally, we compared the results with the detonating failed deflagration model of Kasen \& Plewa (2007) and found that the low continuum polarization but high line polarization are consistent with the model. A viewing angle of $\theta \approx 0^{\circ}$ is favoured, which means we may be viewing SN 2019ein from the ignition side.

We recommend high-quality spectropolarimetric observations of bright, nearby future SNe Ia to be carried out covering both their rising and falling phases. Such polarimetric tomography is essential for building a robust picture of how polarization signatures vary over time and the consequences of various explosion mechanisms and progenitor scenarios.

\section{ACKNOWLEDGEMENTS}

K.C.P. is thankful to Sergiy Vasylyev and Matthew Chu for helpful discussions. A.V.F.'s group at U.C. Berkeley acknowledges generous support from the Miller Institute for Basic Research in Science, Sunil Nagaraj, Landon Noll, Gary and Cynthia Bengier, Clark and Sharon Winslow, Sanford Robertson, and many additional donors. The UCSC team is supported in part by NASA grants NNG17PX03C, 80NSSC19K1386, and 80NSSC20K0953; NSF grant AST-1815935; the Gordon \& Betty Moore Foundation; the Heising-Simons Foundation; and by a fellowship from the David and Lucile Packard Foundation to R.J.F. The research of Y.Y. is supported through the Bengier-Winslow-Robertson Fellowship and the Benoziyo Prize Postdoctoral Fellowship. P.H. acknowledges the support by the NSF project "Signatures of Type Ia Supernovae, New Physics and Cosmology," grant AST-1715133. The supernova research by L.W. is supported by NSF award AST-1817099. J.C.W. is supported by NSF grant AST-1813825. The research of J.M. is supported through a Royal Society University Research Fellowship. M.B. acknowledges support from the Swedish Research Council (Reg. No. 2020-03330).

A major upgrade of the Kast spectrograph on the Shane $3 \mathrm{~m}$ telescope at Lick Observatory, led by Brad Holden, was made possible through generous gifts from the Heising-Simons Foundation, William and Marina Kast, and the University of California Observatories. KAIT and its ongoing operation were made possible by donations from Sun Microsystems, Inc., the Hewlett-Packard Company, AutoScope Corporation, Lick Observatory, the U.S. NSF, the University of California, the Sylvia \& Jim Katzman Foundation, and the TABASGO Foundation. Research at Lick Observatory is partially supported by a generous gift from Google. We appreciate the excellent assistance of the staff at Lick Observatory.

PyRAF, PyFITS, STSCI_PYTHON are products of the Space Telescope Science Institute (STScI), which is operated by AURA for NASA. STScI is operated by the Association of Universities for Research in Astronomy, Inc., under NASA contract NAS5-26555. This research has made use of NASA's Astrophysics Data System Bibliographic Services; the SIMBAD database, operated at CDS, Strasbourg, France; and the NASA/IPAC Extragalactic Database (NED) which is operated by the Jet Propulsion Laboratory, California Institute of Technology, under contract with NASA.

\section{DATA AVAILABILITY}

The raw data used in this work may be shared upon request to Kishore C. Patra (kcpatra@berkeley.edu).

\section{REFERENCES}

Arnett W. D., 1969, AP\&SS, 5, 180

Arnold D. M., Steele I. A., Bates S. D., Mottram C. J., Smith R. J., 2012, in McLean I. S., Ramsay S. K., Takami H., eds, Society of Photo-Optical Instrumentation Engineers (SPIE) Conference Series Vol. 8446, Ground-based and Airborne Instrumentation for Astronomy IV. p. 84462J, doi:10.1117/12.927000

Benz W., Bowers R. L., Cameron A. G. W., Press W. H. ., 1990, ApJ, 348, 647

Bildsten L., Shen K. J., Weinberg N. N., Nelemans G., 2007, ApJL, 662, L95

Bulla M., Sim S. A., Kromer M., 2015, MNRAS, 450, 967

Bulla M., Sim S. A., Pakmor R., Kromer M., Taubenberger S., Röpke F. K., Hillebrandt W., Seitenzahl I. R., 2016a, MNRAS, 455, 1060

Bulla M., et al., 2016b, MNRAS, 462, 1039

Cikota A., et al., 2019, MNRAS, 490, 578

Ciotti L., D’Ercole A., Pellegrini S., Renzini A., 1991, ApJ, 376, 380

Fesen R. A., Höflich P. A., Hamilton A. J. S., Hammell M. C., Gerardy C. L., Khokhlov A. M., Wheeler J. C., 2007, ApJ, 658, 396

Filippenko A. V., 1982, PASP, 94, 715

Filippenko A. V., 1997, ARAA, 35, 309

Filippenko A. V., Li W. D., Treffers R. R., Modjaz M., 2001, in Paczynski B., Chen W.-P., Lemme C., eds, Astronomical Society of the Pacific Conference Series Vol. 246, IAU Colloq. 183: Small Telescope Astronomy on Global Scales. p. 121

Fink M., Röpke F. K., Hillebrandt W., Seitenzahl I. R., Sim S. A., Kromer M., 2010, A\&A, 514, A53

Gal-Yam A., 2017, Observational and Physical Classification of Supernovae. p. 195, doi:10.1007/978-3-319-21846-5_35

Gal-Yam A., et al., 2014, Nature, 509, 471

Gamezo V. N., Khokhlov A. M., Oran E. S., 2004, Physical Review Letters, 92, 211102

García-Berro E., Lorén-Aguilar P., 2017, Dynamical Mergers. p. 1237, doi:10.1007/978-3-319-21846-5_60

García-Berro E., Badenes C., Aznar-Siguán G., Lorén-Aguilar P., 2017, MNRAS, 468, 4815

Hayden B. T., et al., 2010, ApJ, 722, 1691

Hillebrandt W., Kromer M., Röpke F. K., Ruiter A. J., 2013, Frontiers of Physics, 8, 116

Höflich P., 1991, A\&A, 246, 481

Höflich P., 1995, ApJ, 443, 89

Höflich P., 2017, Explosion Physics of Thermonuclear Supernovae and Their Signatures. p. 1151, doi:10.1007/978-3-319-21846-5_56

Höflich P., Khokhlov A., 1996, ApJ, 457, 500

Höflich P., Mueller E., Khokhlov A., 1993, A\&A, 268, 570

Höflich P., Wheeler J. C., Hines D. C., Trammell S. R., 1996, ApJ, 459, 307

Höflich P., Wheeler J. C., Thielemann F. K., 1998, ApJ, 495, 617

Höflich P., Gerardy C. L., Marion H., Quimby R., 2006, New Astronomy Review, 50, 470

Höflich P., et al., 2017, ApJ, 846, 58

Horne K., 1986, PASP, 98, 609

Howell D. A., 2011, Nature Communications, 2, 350

Howell D. A., Höflich P., Wang L., Wheeler J. C., 2001, ApJ, 556, 302

Hoyle F., Fowler W. A., 1960, ApJ, 132, 565

Iben Jr. I., Tutukov A. V., 1984, ApJs, 54, 335

Jermak H., 2017, PhD thesis, Astrophysics Research Institute, Liverpool John Moores University

Kasen D., Plewa T., 2007, ApJ, 662, 459

Kasen D., Thomas R. C., Nugent P., 2006, ApJ, 651, 366

Kasen D., Röpke F. K., Woosley S. E., 2009, Nature, 460, 869

Katz M. P., Zingale M., Calder A. C., Swesty F. D., Almgren A. S., Zhang W., 2016, ApJ, 819, 94

Kawabata M., et al., 2020, ApJ, 893, 143

Khokhlov A. M., 1991, A\&A, 245, 114

Kromer M., Sim S. A., Fink M., Röpke F. K., Seitenzahl I. R., Hillebrandt W., 2010, ApJ, 719, 1067

Kushnir D., Katz B., Dong S., Livne E., Fernández R., 2013, ApJL, 778, L37

Leonard D. C., Filippenko A. V., Chornock R., Li W., 2002, AJ, 124, 2506 
Leonard D. C., Li W., Filippenko A. V., Foley R. J., Chornock R., 2005, ApJ, 632,450

Livne E., 1990, ApJL, 354, L53

Livne E., 1999, ApJL, 527, L97

Luminet J. P., Pichon B., 1989, A\&A, 209, 103

Ma B., Wei P., Shang Z., Wang L., Wang X., 2014, The Astronomer's Telegram, 5794

Maoz D., Mannucci F., Nelemans G., 2014, ARAA, 52, 107

Maund J. R., et al., 2013, MNRaS, 433, L20

Maund J. R., et al., 2021, MNRAS, 503, 312

Miller J. S., Robinson L. B., Goodrich R. W., 1988, in Instrumentation for Ground-Based Optical Astronomy. p. 157

Moll R., Raskin C., Kasen D., Woosley S. E., 2014, ApJ, 785, 105

Niemeyer J. C., Hillebrandt W., Woosley S. E., 1996, ApJ, 471, 903

Nomoto K., 1982a, ApJ, 253, 798

Nomoto K., 1982b, ApJ, 257, 780

Nomoto K., Sugimoto D., Neo S., 1976, APSS, 39, L37

Nomoto K., Thielemann F.-K., Yokoi K., 1984, ApJ, 286, 644

Nugent P., Baron E., Branch D., Fisher A., Hauschildt P. H., 1997, ApJ, 485, 812

Nugent P. E., et al., 2011, Nature, 480, 344

Pakmor R., Kromer M., Röpke F. K., Sim S. A., Ruiter A. J., Hillebrandt W., 2010, Nature, 463, 61

Pakmor R., Hachinger S., Röpke F. K., Hillebrandt W., 2011, A\&A, 528, A117

Pakmor R., Kromer M., Taubenberger S., Sim S. A., Röpke F. K., Hillebrandt W., 2012, ApJL, 747, L10

Patat F., Baade D., Höflich P., Maund J. R., Wang L., Wheeler J. C., 2009, A\&A, 508, 229

Pellegrino C., et al., 2020, ApJ, 897, 159

Perlmutter S., et al., 1999, ApJ, 517, 565

Phillips M. M., 1993, ApJL, 413, L105

Plewa T., Calder A. C., Lamb D. Q., 2004, ApJL, 612, L37

Raskin C., Kasen D., Moll R., Schwab J., Woosley S., 2014, ApJ, 788, 75

Reinecke M., Hillebrandt W., Niemeyer J. C., 2002, A\&A, 391, 1167

Renzini A., 1999, in Walsh J. R., Rosa M. R., eds, Chemical Evolution from Zero to High Redshift. p. 185 (arXiv: astro-ph/9902361)

Riess A. G., 2020, Nature Reviews Physics, 2, 10

Riess A. G., et al., 1998, AJ, 116, 1009

Röpke F. K., 2007, ApJ, 668, 1103

Rosswog S., Ramirez-Ruiz E., Hix W. R., 2009, ApJ, 695, 404

Sato Y., Nakasato N., Tanikawa A., Nomoto K., Maeda K., Hachisu I., 2016, ApJ, 821, 67

Schlafly E. F., Finkbeiner D. P., 2011, ApJ, 737, 103

Schmidt G. D., Elston R., Lupie O. L., 1992, AJ, 104, 1563

Seitenzahl I. R., et al., 2013, MNRAS, 429, 1156

Serkowski K., Mathewson D. S., Ford V. L., 1975, ApJ, 196, 261

Shen K. J., Bildsten L., 2009, ApJ, 699, 1365

Shen K. J., Kasen D., Weinberg N. N., Bildsten L., Scannapieco E., 2010, ApJ, 715, 767

Shen K. J., Kasen D., Miles B. J., Townsley D. M., 2018, ApJ, 854, 52

Soker N., 2019, New Astron. Rev., 87, 101535

Stevance H. F., et al., 2019, MNRAS, 485, 102

Taam R. E., 1980, ApJ, 242, 749

Tinyanont S., et al., 2021, Nature Astronomy, 5, 544

Tonry J., et al., 2019, Transient Name Server Discovery Report, 2019-3, 1

Wang L., Wheeler J. C., 2008, ARAA, 46, 433

Wang L., Wheeler J. C., Höflich P., 1997, ApJL, 476, L27

Wang L., Howell D. A., Höflich P., Wheeler J. C., 2001, ApJ, 550, 1030

Wang L., et al., 2003, ApJ, 591, 1110

Wang L., Baade D., Höflich P., Wheeler J. C., Kawabata K., Khokhlov A., Nomoto K., Patat F., 2006, ApJ, 653, 490

Wang L., Baade D., Patat F., 2007, Science, 315, 212

Wang X., Li W., Filippenko A. V., Foley R. J., Smith N., Wang L., 2008, ApJ, 677, 1060

Webbink R. F., 1984, ApJ, 277, 355

Whelan J., Iben Jr. I., 1973, ApJ, 186, 1007

Wolff M. J., Nordsieck K. H., Nook M. A., 1996, AJ, 111, 856
Woosley S. E., Weaver T. A., 1994, ApJ, 423, 371

Woosley S. E., Weaver T. A., Taam R. E., 1980, in Wheeler J. C., ed., Texas Workshop on Type I Supernovae. pp 96-112

Yang Y., et al., 2020, ApJ, 902, 46

van Dokkum P. G., 2001, PASP, 113, 1420

This paper has been typeset from a $\mathrm{T}_{\mathrm{E}} \mathrm{X} / \mathrm{L} \mathrm{T} \mathrm{E} \mathrm{X}$ file prepared by the author. 